\title{
Microfluidics-Based Biochips: Technology Issues, Implementation Platforms, and Design-Automation Challenges
}

\author{
Fei Su, Student Member, IEEE, Krishnendu Chakrabarty, Senior Member, IEEE, and Richard B. Fair, Fellow, IEEE
}

\begin{abstract}
Microfluidics-based biochips are soon expected to revolutionize clinical diagnosis, deoxyribonucleic acid (DNA) sequencing, and other laboratory procedures involving molecular biology. In contrast to continuous-flow systems that rely on permanently etched microchannels, micropumps, and microvalves, digital microfluidics offers a scalable system architecture and dynamic reconfigurability; groups of unit cells in a microfluidics array can be reconfigured to change their functionality during the concurrent execution of a set of bioassays. As more bioassays are executed concurrently on a biochip, system integration and design complexity are expected to increase dramatically. This paper presents an overview of an integrated system-level design methodology that attempts to address key issues in the synthesis, testing and reconfiguration of digital microfluidics-based biochips. Different actuation mechanisms for microfluidics-based biochips, and associated design-automation trends and challenges are also discussed. The proposed top-down design-automation approach is expected to relieve biochip users from the burden of manual optimization of bioassays, time-consuming hardware design, and costly testing and maintenance procedures, and it will facilitate the integration of fluidic components with a microelectronic component in next-generation systems-on-chips (SOCs).
\end{abstract}

Index Terms-Biochips, design automation, microfluidics, reconfiguration, synthesis, testing.

\section{INTRODUCTION}

$\mathbf{M}$ ICROFLUIDICS-BASED biochips for biochemical analysis are receiving much attention nowadays [1]-[4]. These composite microsystems, also known as lab-on-a-chip or bio-microelectromechanical system (MEMS), offer a number of advantages over conventional laboratory procedures. They automate highly repetitive laboratory tasks by replacing cumbersome equipment with miniaturized and integrated systems, and they enable the handling of small amounts, e.g., micro- and nanoliters, of fluids. Thus, they are able to provide ultrasensitive detection at significantly lower costs per assay than traditional methods, and in a significantly smaller amount of laboratory space.

Advances in microfluidics technology offer exciting possibilities in the realm of enzymatic analysis (e.g., glucose and

Manuscript received February 23, 2005; revised May 16, 2005. This work was supported by the National Science Foundation under Grant IIS-0312352. This paper was recommended by Associate Editor J. Zeng.

The authors are with the Department of Electrical and Computer Engineering, Duke University, Durham, NC 27708 USA (e-mail: fs@ee.duke.edu; krish@ee.duke.edu; rfair@ee.duke.edu).

Digital Object Identifier 10.1109/TCAD.2005.855956 lactate assays), deoxyribonucleic acid (DNA) analysis [e.g., polymerase chain reaction (PCR) and nucleic-acid sequence analysis], proteomic analysis involving proteins and peptides, immunoassays, and toxicity monitoring. An emerging application area for microfluidics-based biochips is clinical diagnostics, especially immediate point-of-care diagnosis of diseases [5], [6]. Microfluidics can also be used for countering bioterrorism threats [7], [8]. Microfluidics-based devices, capable of continuous sampling and real-time testing of air/water samples for biochemical toxins and other dangerous pathogens, can serve as an always-on "bio-smoke alarm" for early warning.

The first generation of microfluidic biochips contained permanently etched micropumps, microvalves, and microchannels, and their operation was based on the principle of continuous fluid flow [3], [4]. A promising alternative is to manipulate liquids as discrete droplets [9], [10]. Following the analogy of microelectronics, this novel approach is referred to as "digital microfluidics." In contrast to continuous-flow biochips, digital microfluidics-based biochips, which we also refer to as secondgeneration biochips, offer scalable system architecture based on a two-dimensional (2-D) microfluidic array of identical basic unit cells. Moreover, because each droplet can be controlled independently, these "digital" systems also have dynamic reconfigurability, whereby groups of unit cells in a microfluidic array can be reconfigured to change their functionality during the concurrent execution of a set of bioassays. The advantages of scalability and reconfigurability make digital microfluidic biochips a promising platform for massively parallel DNA analysis, automated drug discovery, and real-time biomolecular detection.

As the use of digital microfluidics-based biochips increases, their complexity is expected to become significant due to the need for multiple and concurrent assays on the chip, as well as more sophisticated control for resource management. Timeto-market and fault tolerance are also expected to emerge as design considerations. As a result, current full-custom design techniques will not scale well for larger designs. There is a pressing need to deliver the same level of computeraided design (CAD) support to the biochip designer that the semiconductor industry now takes for granted. Moreover, it is expected that these microfluidic biochips will be integrated with microelectronic components in next-generation system-on-chip (SOC) designs. The 2003 International Technology Roadmap for Semiconductors (ITRS) clearly identifies the integration of electrochemical and electrobiological techniques as one of the 
system-level design challenges that will be faced beyond 2009, when feature sizes shrink below $50 \mathrm{~nm}$ [11].

As digital microfluidics-based biochips become widespread in safety-critical biochemical applications, the reliability of these systems will emerge as a critical performance parameter. These systems need to be tested adequately not only after fabrication, but also continuously during in-field operation. For instance, for detectors monitoring for dangerous pathogens in critical locations such as airports, field testing is critical to ensure low false-positive and false-negative detection rates. In such cases, concurrent testing, which allows testing and normal bioassays to run simultaneously on a microfluidic system, can play an important role. It consequently facilitates built-in self-test (BIST) of digital microfluidic biochips and makes them less dependent on costly manual maintenance on a regular basis. Therefore, there exists a need for efficient testing methodologies for these microsystems. Due to the underlying mixed technology and multiple energy domains, the microfluidic biochip exhibits unique failure mechanisms and defects. In fact, the ITRS 2003 document recognizes the need for new test methods for disruptive device technologies that underlie microelectromechanical systems and sensors, and highlights it as one of the five difficult test challenges beyond 2009 [11].

The reconfigurability inherent in digital microfluidic biochips can be utilized to achieve longer system lifetime through on-line reconfiguration to avoid operational faults. It can also be used to increase production yield through production-time reconfiguration to bypass manufacturing faults. System reliability motivates the need for on-line reconfiguration techniques to tolerate faults during field operation. Reconfiguration is also useful for yield enhancement because it can be used to tolerate manufacturing faults. In this scenario, we assume that a microfluidic biochip has been fabricated for a set of bioassays, but some defective unit cells are identified prior to its deployment. The configuration of the microfluidic array must therefore be changed in such a way that the functionality of the bioassays is not compromised.

In this paper, we present an overview of an integrated methodology that addresses key issues in the synthesis, testing, and reconfiguration of digital microfluidic biochips. The goal here is to provide top-down system-level design-automation tools to biochip users, which can relieve them from the burden of manual optimization of assays, time-consuming hardware design, and costly testing and maintenance procedures. Users will be able to describe bioassays at a sufficiently high level of abstraction; synthesis tools will then map the behavioral description to a microfluidic biochip and generate an optimized schedule of bioassay operations, the binding of assay operations to resources, and a layout of the microfluidic biochip. For fabricated microfluidic biochips, cost-effective testing techniques will be available to detect faulty unit cells after the manufacture and during field operation. On-line and off-line reconfiguration techniques, incorporated in these design-automation tools, will then be used to easily bypass faulty unit cells once they are detected, and remap bioassays operations to other fault-free resources, thereby supporting defect/fault tolerance. Thus, the biochip user can concentrate on the development of the nanoand microscale bioassays, leaving implementation details to the design-automation tools. Therefore, these tools will reduce human effort and enable high-volume production.

The organization of the remainder of the paper is as follows. Section II reviews biochip and microfluidics technology. Different actuation mechanisms for microfluidics-based biochips are discussed. We also present an overview of digital microfluidic biochips based on electrowetting. Next, Section III discusses design trends and challenges for digital microfluidics-based biochips. After reviewing today's design techniques, we propose a top-down design methodology for digital microfluidic biochips. This methodology encompasses synthesis, testing, and reconfiguration. Challenges in the proposed system-level design method are also identified and discussed. Finally, conclusions are drawn in Section IV.

\section{BiochiP AND MicRofluidics TECHNOLOGY}

\section{A. Biochips}

Early biochips were based on the concept of a DNA microarray, which is a piece of glass, plastic, or silicon substrate on which pieces of DNA have been affixed in a microscopic array. Scientists use such chips to screen a biological sample simultaneously for the presence of many genetic sequences at once. The affixed DNA segments are known as probes. Thousands of identical probe molecules are affixed at each point in the array to make the chips effective detectors. The flowchart of DNA microarray production and operation is shown in Fig. 1. Note that sample preparation need to be carried out off chip. There are a number of commercial microarrays available in the market place such as GeneChip DNAarray from Affymetrix, DNA microarray from Infineon AG, or NanoChip microarray from Nanogen [12]-[14]. Similar to a DNA microarray, a protein array is a miniature array where a multitude of different capture agents, most frequently monoclonal antibodies, are deposited on a chip surface (glass or silicon); they are used to determine the presence and/or amount of proteins in biological samples, e.g., blood. A drawback of DNA and protein arrays is that they are neither reconfigurable nor scalable after manufacture. Moreover, they lack the ability to carry out sample preparation, which is critical to biochemical applications.

The basic idea of microfluidic biochips is to integrate all necessary functions for biochemical analysis onto one chip using microfluidics technology. These micro-total-analysis-systems ( $\mu$ TAS) are more versatile and complex than microarrays. Integrated functions include microfluidic assay operations and detection, as well as sample pretreatment and preparation. So far, there are two different generations of microfluidic biochips, namely continuous-flow biochips and droplet-based microfluidic biochips.

\section{B. Microfluidics}

1) Continuous-Flow Microfluidics: The first generation microfluidic technologies are based on the manipulation of continuous liquid flow through microfabricated channels. Actuation of liquid flow is implemented either by external pressure sources, integrated mechanical micropumps, or by electrokinetic mechanisms [3], [4]. For example, 


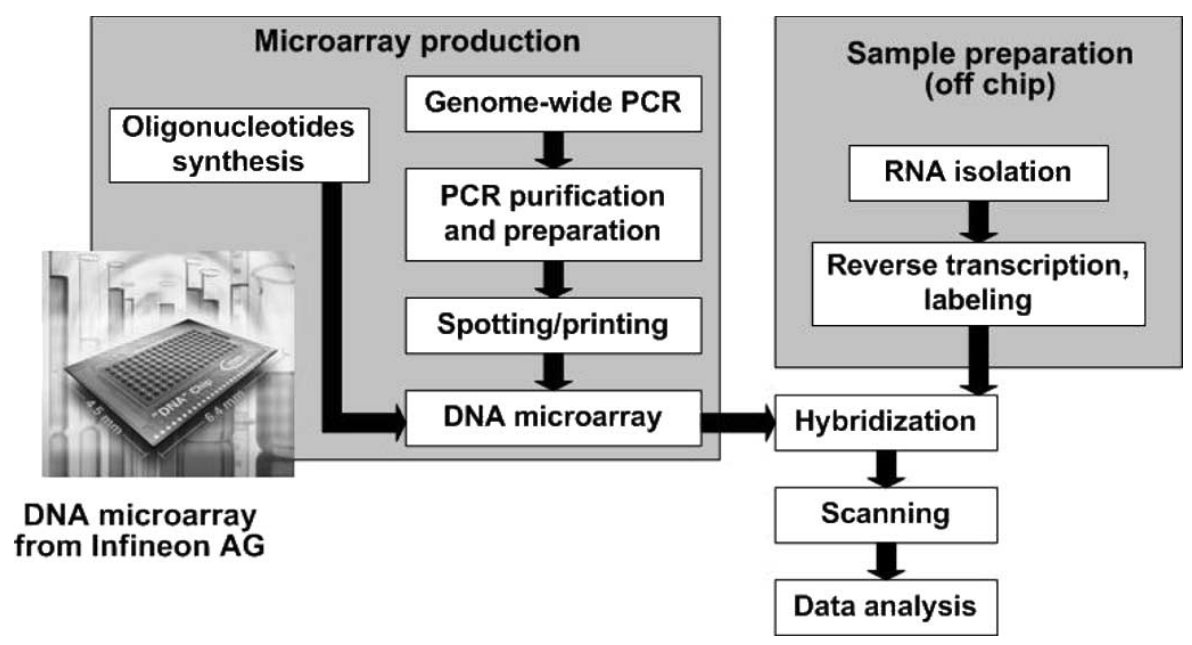

Fig. 1. Steps in the production and operation of a DNA microarray.

electroosmosis is a commonly used electrokinetic method, which refers to the motion of an ionic fluid solution by means of an electrical field. As shown in Fig. 2(a), a double layer of ions, consisting of a compact immobile layer and a mobile diffuse layer, is formed in the liquid sandwiched between two glass plates [15]. If an electric field is applied parallel to the liquid-solid interface, mobile charges in the diffuse layers are moved, consequently dragging the liquid with them. Fig. 2(b) demonstrates the forward and reverse liquid flow in a fabricated microchannel when forward and reversed dc voltages are applied, respectively; this continuous-flow microfluidic system based on electroosmosis was developed at the University of Michigan [15].

Continuous-flow systems are adequate for many well-defined and simple biochemical applications, but they are unsuitable for more complex tasks requiring a high degree of flexibility or complicated fluid manipulations [3], [4]. These closed-channel systems are inherently difficult to integrate and scale because the parameters that govern the flow field (e.g., pressure, fluid resistance, and electric field) vary along the flow path making the fluid flow at any one location dependent on the properties of the entire system. Moreover, unavoidable shear flow and diffusion in microchannels makes it difficult to eliminate intersample contamination and dead volumes. Permanently etched microstructures also lead to limited reconfigurability and poor fault-tolerance capability. Therefore, the fabrication of complex yet reliable continuous-flow biochips remains a major technical challenge.

2) Droplet-Based Microfluidics: Alternatives to the above closed-channel continuous-flow systems include novel open structures, where the liquid is divided into discrete independently controllable droplets, and these droplets can be manipulated to move on a substrate [9], [10], [22]. By using discrete unit-volume droplets, a microfluidic function can be reduced to a set of repeated basic operations, i.e., moving one unit of fluid over one unit of instance. This "digitization" method facilitates the use of a hierarchical and cell-based approach for microfluidic biochip design. In this scenario, we envisage that a large-scale integrated digital microfluidic biochip can be constructed out of repeated instances of well-characterized unit

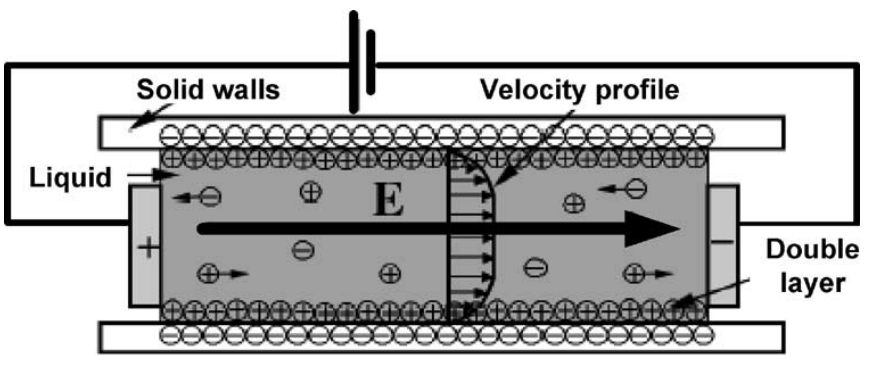

(a)

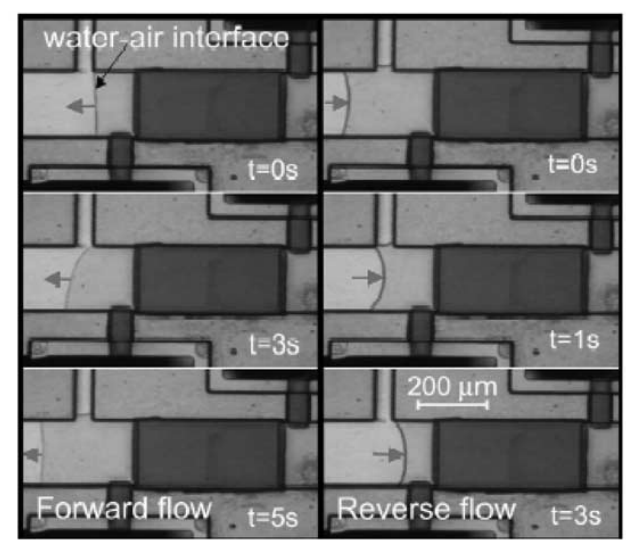

(b)

Fig. 2. (a) Depiction of electroosmotic flow; (b) forward and reverse fluid flow with dc voltages applied and polarities reversed, respectively [15].

cells in the same way that complex very-large-scale-integration (VLSI) circuits may be built upon well-characterized transistors. Moreover, the constituent microfluidic unit cells, referred to as microfluidic modules, can be reorganized at different levels of hierarchy to support biochemical applications of various scales. Defect/fault tolerance is also easily incorporated in the design due to the inherent dynamic reconfigurability. Therefore, in contrast to continuous fluid flow, digital microfluidics offer a flexible and scalable system architecture as well as high defect/tolerance capability.

A number of methods for manipulating microfluidic droplets have been proposed in the literature [16]-[18], [20]-[22]. These 

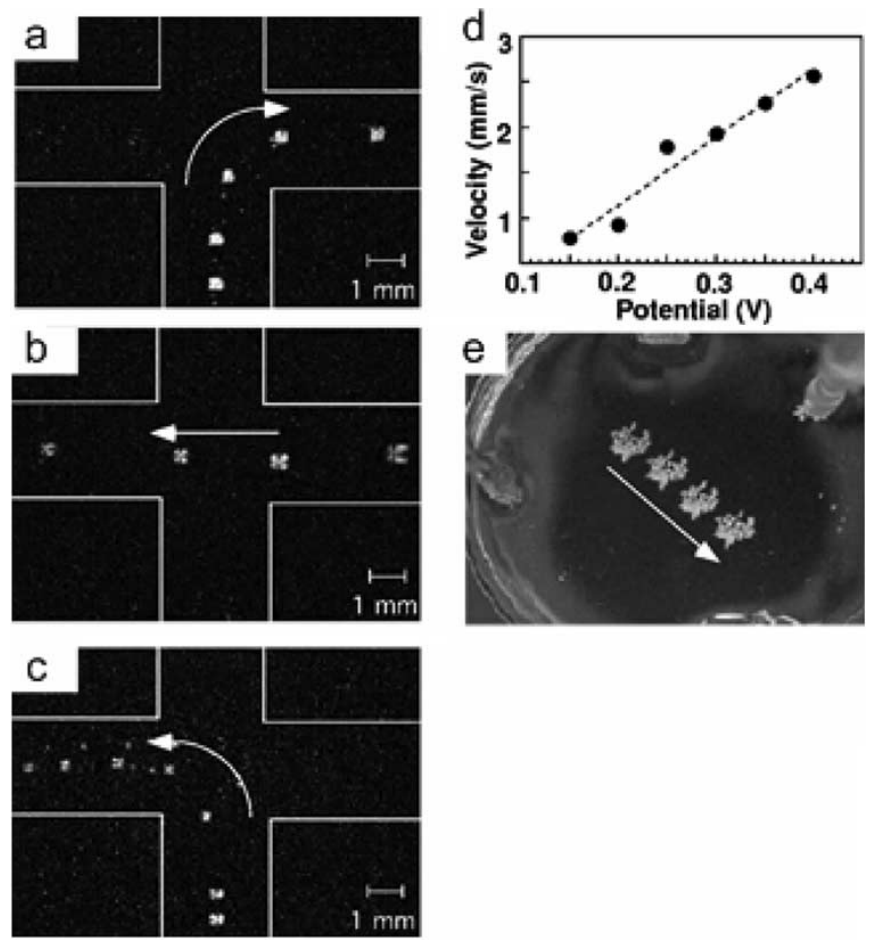

Fig. 3. (a)-(c) Movement of LC droplets through a simple fluidic network; (d) movement velocity as a function of the applied potential; (e) movement of sulfur microparticles [16].

techniques can be classified as chemical, thermal, acoustical, and electrical methods. For example, Gallardo et al. proposed an electrochemical method, whereby they used a voltage-controlled, reversible electrochemical reaction that creates or consumes redox-active surfactants (i.e., surface-active molecules). This reaction generates a surface-tension gradient along a channel [16]. The surface-tension gradient is capable of driving liquid droplets through a simple fluidic network; an example is shown in Fig. 3 [16]. Time-lapse images in Fig. 3(a)-(c) demonstrate the movement of liquid crystal (LC) droplets based on the electrochemical method. As shown in Fig. 3(d), the velocity of fluid motion is a function of the applied potential; moderate velocities of $2.5 \mathrm{~mm} / \mathrm{s}$ were obtained at low voltages $(<1 \mathrm{~V})$. Fig. 3(e) also illustrates the image of the transportation of sulfur microparticles across the surface of an aqueous solution. However, since the electrochemical gradient must be established along the entire length of the channel, this technique, like electrokinetic methods used in continuous-flow systems, does not provide a convenient way to independently control multiple droplets.

In another electrochemical method, Ichimura et al. used a photoresponsive surface to generate surface-energy gradients to drive droplets [17]. Photographs of light-driven motion of an olive-oil droplet on a silica plate, which is modified with macrocyclic-amphiphile-tethering photochromic azobenzene units, are shown in Fig. 4 [17]. However, the reported dropletmovement velocities of $50 \mu \mathrm{m} / \mathrm{s}$ are very slow and many liquids including water cannot be transported by this technique due to contact-angle hysteresis.

Another type of effect, namely thermocapillarity, exploits the temperature dependence of surface tension to drive droplet mo-
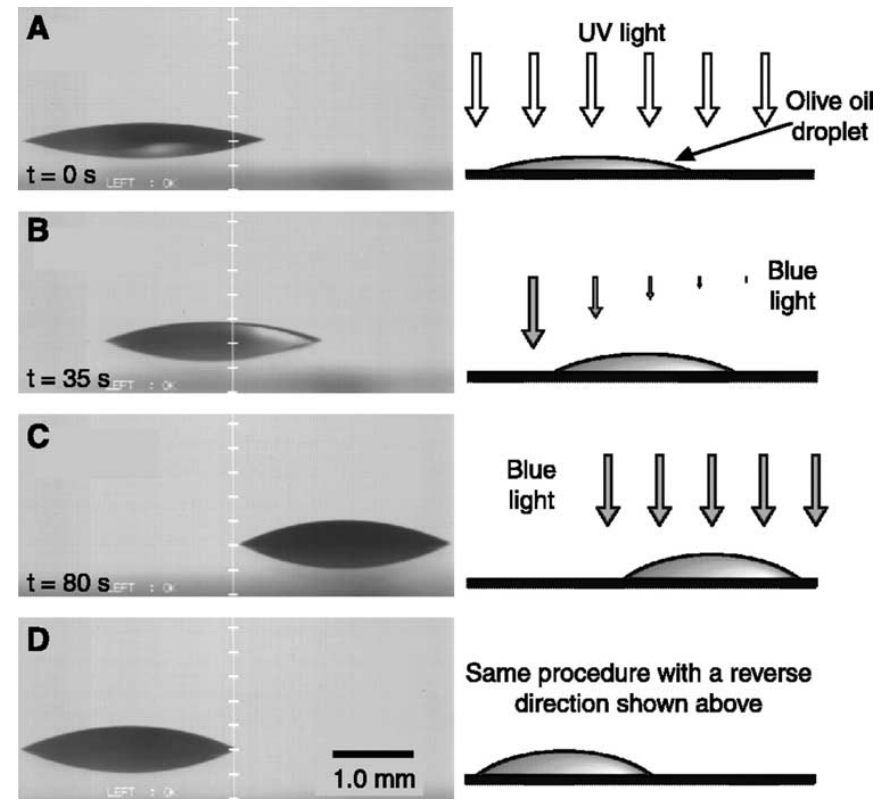

Fig. 4. Lateral photographs of light-driven motion of an olive-oil droplet on a silica plate by asymmetrical irradiation with 436-nm light perpendicular to the surface [17].

tion [18]. Thermocapillarity-based systems incorporate multiple independently controllable micromachined heaters into a substrate to control multiple droplets. However, the design and analysis of these systems is complex due to the critical requirement of complete and complicated heat-transfer analysis. Moreover, to achieve a modest velocity (e.g., $20 \mathrm{~mm} / \mathrm{s}$ ), a relatively high temperature gradient (e.g., a differential of $20-40{ }^{\circ} \mathrm{C}$ ) is needed. Unfortunately, such large temperature variations are unacceptable for many biochemical applications where temperature control to within the $1{ }^{\circ} \mathrm{C}$ range is desired [19].

Surface acoustic waves (SAW) can be used to propagate across the piezoelectric substrate just like earthquakes do, driving droplets to move on the chip surface, as shown in Fig. 5 [20]. Given the right frequency of the signal, a mechanical wave is launched across the chip; the forces within this "nanoearthquake" are sufficient to actuate the droplet on the surface. SAW-based technology can also be used to perform droplet mixing. At low power levels, the SAW is converted into an internal streaming in the droplet. In contrast to the diffusion, streaming induces a very efficient mixing and stirring within the droplet. Furthermore, if a different frequency is applied during this process, different streaming patterns are induced and superimposed, leading to quasi-chaotic mixing [20].

In addition to the above chemical and thermal methods, electrical methods to actuate droplets have received considerable attention in recent years [9], [10], [21]-[23]. Dielectrophoresis (DEP) and electrowetting-on-dielectric (EWOD) are the two most common electrical methods. DEP relies on the application of high-frequency ac voltages [22], [23], while EWOD is based on dc (or low-frequency ac) voltages [9], [10]. Both these techniques take advantage of electrohydrodynamic forces, and they can provide high droplet speeds with relatively simple geometries. Liquid DEP actuation is defined as the attraction 

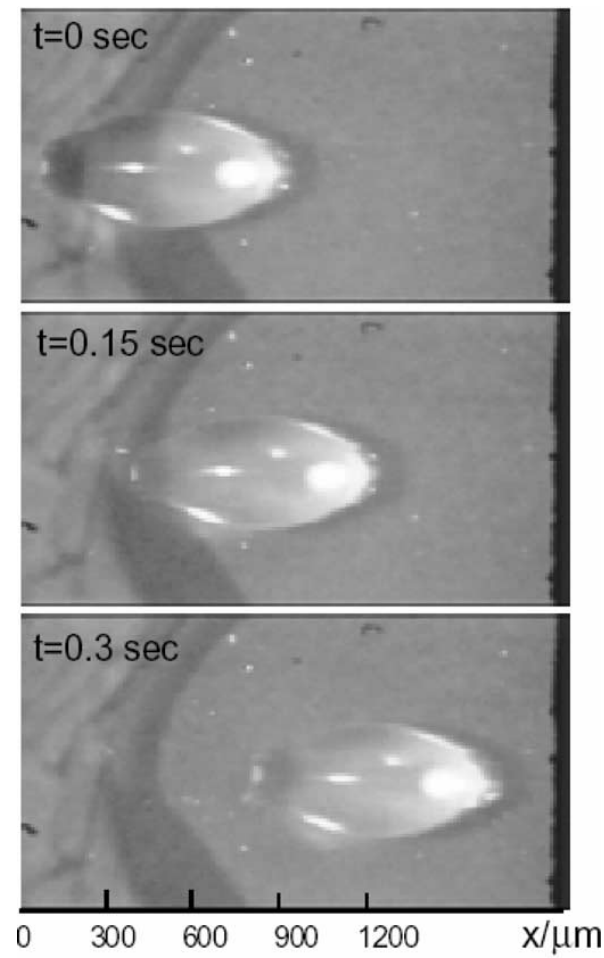

Fig. 5. Photos of droplet motion caused by SAW forces [20].

of polarizable liquid masses into the regions of higher electric-field intensity, as shown in Fig. 6 [24]. DEP-based microfluidics relies on coplanar electrodes patterned on a substrate, coated with a thin dielectric layer, and energized with ac voltage (200-300 V · rms at 50-200 kHz). Rapid dispensing of large numbers of picoliter-volume droplets and a voltagecontrolled array mixer have been demonstrated using DEP [22]. Images of multiple droplet movement on an $8 \times 82$-D electrode array driven by DEP forces are shown in Fig. 7 [23]; this DEP-driven microfluidic array was developed at the University of Texas M. D. Anderson Cancer Center. However, excessive Joule heating may be a problem for DEP actuation, even though it can be reduced by using materials of higher thermal conductivity or by reducing structure size [22], [25]. EWOD uses dc (or low-frequency ac) electric fields to directly control the interfacial energy between a solid and liquid phase. In contrast to DEP actuation, Joule heating is virtually eliminated in EWOD because the dielectric layer covering the electrodes blocks dc electric current. As a consequence, aqueous solutions with salt concentration as high as $0.15 \mathrm{M}$ can be actuated without heating [25]. The EWOD technique for digital microfluidic biochips forms the basis of the work reported in this paper; we describe it in more detail in the next section.

3) Digital Microfluidics-Based Biochips: The digital microfluidic biochips discussed in this paper are based on the manipulation of nanoliter droplets using the principle of electrowetting. EWOD refers to the modulation of the interfacial tension between a conductive fluid and a solid electrode coated with a dielectric layer by applying an electric field between them. An imbalance of interfacial tension is created if an electric field is applied to only one side of the droplet; this interfacial tension gradient forces the droplet to move.

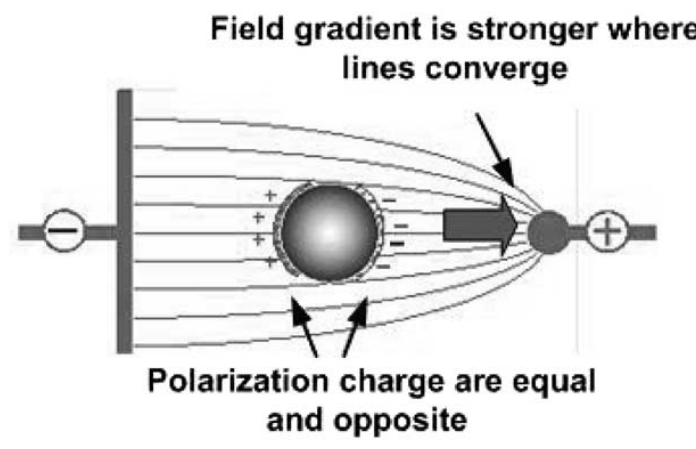

Polarizable droplet in an inhomogeneous electrical field

Fig. 6. Liquid DEP actuation [24].

The basic unit cell of an EWOD-based digital microfluidic biochip consists of two parallel glass plates, as shown in Fig. 8(a). The bottom plate contains a patterned array of individually controllable electrodes, and the top plate is coated with a continuous ground electrode. All electrodes are formed by indium tin oxide (ITO). A dielectric insulator, e.g., parylene $\mathrm{C}$, coated with a hydrophobic film of Teflon AF, is added to the plates to decrease the wettability of the surface and to add capacitance between the droplet and the control electrode. The detailed fabrication process is described in [26]. The droplet containing biochemical samples and the filler medium, such as the silicone oil, are sandwiched between the plates; the droplets travel inside the filler medium. In order to move a droplet, a control voltage is applied to an electrode adjacent to the droplet and at the same time the electrode just under the droplet is deactivated. The EWOD effect causes an accumulation of charge in the droplet/insulator interface, resulting in a interfacial tension gradient across the gap between the adjacent electrodes, which consequently causes the transportation of the droplet. By varying the electrical potential along a linear array of electrodes, electrowetting can be used to move nanolitervolume liquid droplets along this line of electrodes [26]. The velocity of the droplet can be controlled by adjusting the control voltage (0-90 V), and droplets can be moved at speeds of up to $20 \mathrm{~cm} / \mathrm{s}$ [27]. Droplets can also be transported, in user-defined patterns and under clocked-voltage control, over a 2-D array of electrodes shown in Fig. 8(b), without the need for micropumps and microvalves. In the remainder of this paper, EWOD-based digital microfluidic biochips are simply referred to as "digital microfluidic biochips."

The in vitro measurement of glucose and other metabolites, such as lactate, glutamate, and pyruvate, is of great importance in clinical diagnosis of metabolic disorders. A colorimetric enzyme-kinetic glucose assay has been recently demonstrated in lab experiments on a digital microfluidic biochip [6], [28], [29]. This biochip uses a digital microfluidic array, which moves and mixes droplets containing biochemical samples and reagents, and an integrated optical-detection system consisting of a light-emitting diode (LED) and a photodiode; see Fig. 9 [6], [28], [29].

In addition to glucose assays, the detection of other metabolites such as lactate, glutamate, and pyruvate in a digital microfluidics-based biochip has also been demonstrated 


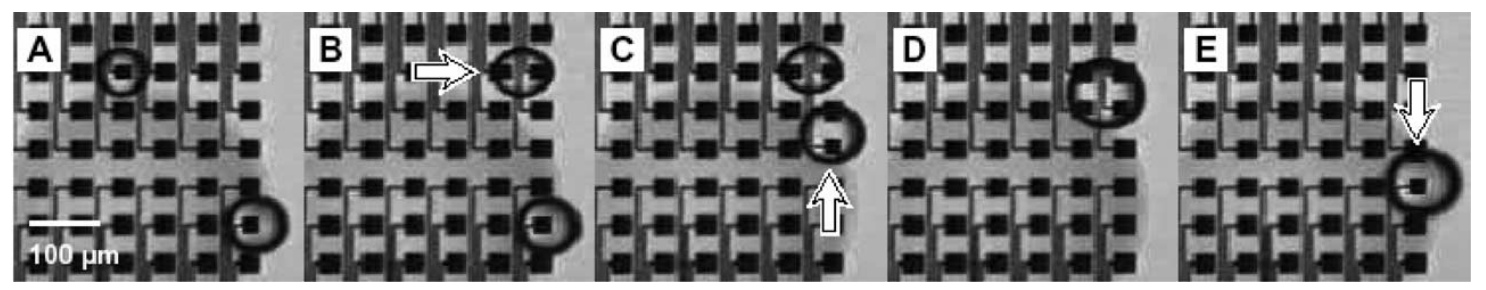

Fig. 7. Droplets are driven by DEP forces on the surface of a 2-D array [23].

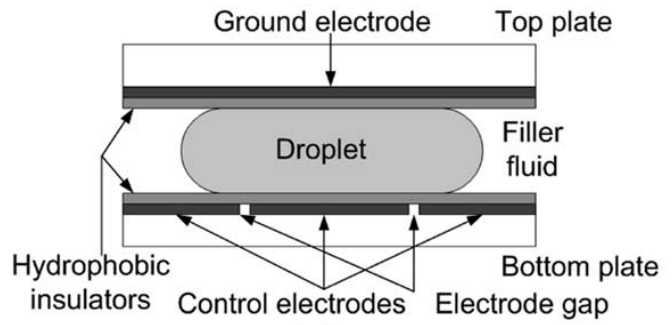

(a)

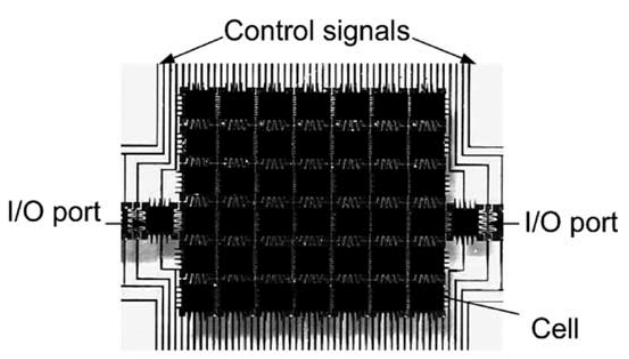

(b)

Fig. 8. (a) Basic unit cell used in an EWOD-based digital microfluidic biochip; (b) a 2-D array for digital microfluidics.

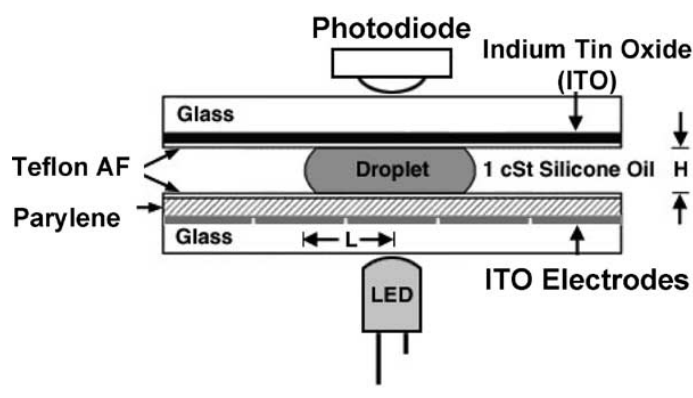

(a)

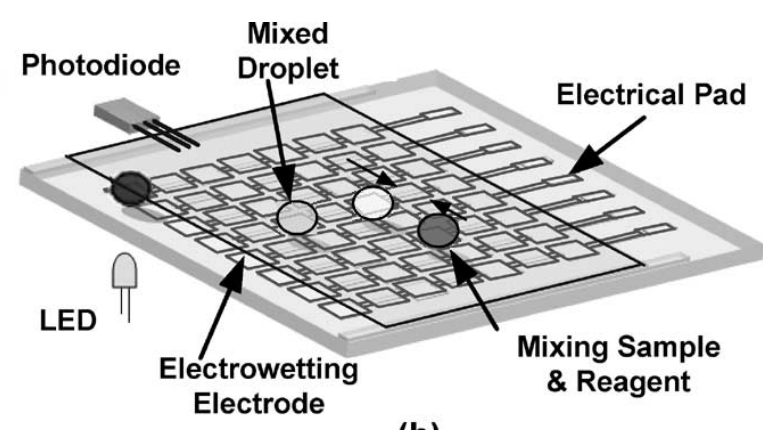

(b)

Fig. 9. Schematic of a digital microfluidic biochip used for colorimetric assays: (a) basic unit cell; (b) top view of microfluidic array.

recently [6], [28], [29]. Furthermore, these assays as well as the glucose assay can be integrated to form a set of multiplexed bioassays that are performed concurrently on a microfluidic platform. Fig. 10 illustrates a fabricated microfluidic system used for multiplexed bioassays [6]. For example, Sample1 contains glucose and Reagent 1 contains glucose oxidase and other chemicals. Similarly, Sample2 contains lactate and Reagent2 consists of lactate oxidase and other chemicals. In this way, both the glucose assay and lactate assay can be carried out concurrently. To demonstrate multiplexed assays, only unit cells and electrodes used for the bioassay have been fabricated. Note however that assays involving whole blood cells have not yet been successfully demonstrated by electrowetting [30]. Despite these limitations, advances in design-automation tools will allow the design and fabrication of generic microfluidic platforms to which a set of assays can be mapped for optimized throughput, resource utilization, and fault tolerance.

\section{Design Trends And Challenges}

\section{A. Typical Design Methodology: Bottom-Up}

MEMS design is a relatively young field compared to integrated-circuit design. Since the concept of special CAD

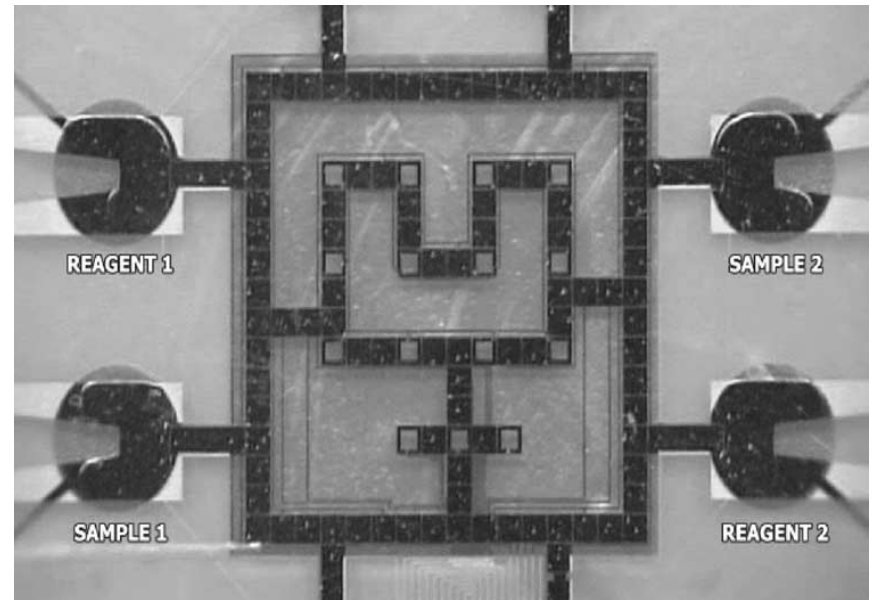

Fig. 10. Fabricated microfluidic array used for multiplexed bioassays [6].

systems for MEMS was first proposed at Transducer'87 [31], several research groups have reported significant progress in this area, and a number of commercial MEMS CAD tools are now available [32], [33]. Many of these tools are focused solely on the modeling of thermal and electro/mechanical properties. Recently, synthesis tools for MEMS have also 
been developed [34]. However, because of the differences in actuation methods between MEMS and microfluidics, they cannot be directly used for the design of microfluidic biochips. While MEMS design tools have reached a certain level of maturity, CAD tools for biochips are still in their infancy. Some design-automation techniques have been proposed for DNA probe arrays [35]; however, as indicated in Section II-A, microfluidics-based biochips are more versatile and complex than DNA arrays.

Current design methodologies for microfluidics-based biochips are typically full custom and bottom up in nature. Since much microfluidics work to date has been focused on device development, most design-automation research for microfluidic biochips has been limited to device-level physical modeling of components [36]-[38]. For example, a combined circuit/device model for the analysis of microfluidic devices that incorporate fluidic transport, chemical reaction, and reagent mixing and separation is presented in [36]. In the proposed circuit/device model, the continuous fluidic network is represented by a circuit model and the functional units of the microfluidic system are represented by appropriate device models. In addition, there are also some available commercial computational-fluid-dynamics (CFD) tools, such as CFDACE+ from CFD Research Corporation and FlumeCAD from Coventor, Inc. that support the three-dimensional (3-D) simulation of microfluidic transport. Recently, physical modeling for digital microfluidics-based biochip has begun to receive much attention [37], [38]. For example, a unified framework of droplet electrohydrodynamics (EHD) to analyze the two major operating principles of droplet-based microfluidics, i.e., DEP and EWOD, is presented in [38]. The numerical simulations based on droplet EHD are validated against analytical and experimental results, and they are then used to illustrate the operation of digital microfluidics-based devices.

Once the devices are optimized using detailed physical simulation, they can be used to assemble a complete microfluidicsbased biochip. Therefore, a bottom-up development approach is rather natural, which involves the development of each block from the device to the system level. Microfluidic devices (e.g., electrodes and glass plates) are combined to form microfluidic modules (e.g., mixers or storage units), which are then combined to obtain the complete system (e.g., microfluidicsbased glucose detectors). Since the system behavior can only be verified at this stage, a costly and time-consuming redesign effort is required at the circuit level if the system does not satisfy design constraints.

Although these full-custom and bottom-up methodologies have been employed successfully in the past, they are clearly inadequate for the design of complex microfluidics-based biochips. As developments in microfluidics continue, it is likely that future microfluidics-based biochips will contain more than hundreds or thousands of basic components. Thus, an efficient design methodology and framework are required. While top-down system-level design tools are now commonplace in integrated-circuit design, few such efforts have been reported for digital microfluidics-based biochips. A recent release of CoventorWare from Coventor, Inc., includes microfluidic behavioral models to allow top-down system-level design [39].
However, this CAD tool is only able to deal with continuousflow systems, and it is therefore inadequate for the design of digital microfluidic biochips.

\section{B. Proposed Design Methodology: Top-Down}

1) Overview: Motivated by the analogy between digital microfluidics-based biochips and digital integrated circuits, we aim to leverage advances in classical integrated circuit CAD techniques to address the design challenges associated with large-scale biochemical applications. The proposed systemlevel top-down design methodology is not only used to reduce biochip design complexity and time-to-market with the aid of design-automation tools, but it can also be extended to enhance yield and system reliability.

The framework of this design methodology is illustrated in Fig. 11. First, the biochip users (e.g., biochemists) provide the protocol for nano- and microscale bioassays. We anticipate that advances in microscale chemistry will lead to such well-defined protocols. A sequencing graph $G(V, E)$ can directly be applied to describe this assay protocol, where vertex set $V=\left\{v_{i}: i=\right.$ $0,1, \ldots, k\}$ in one-to-one correspondence with the set of assay operations and edge set $E=\left\{\left(v_{i}, v_{j}\right): i, j=0,1, \ldots, k\right\}$ represents dependencies between assay operations. We can also use a high-level description language such as SystemC to model the protocol, and then derive a sequencing graph model from it. Moreover, this model can be used to perform behavioral-level simulation to verify the assay functionality at the high level [2]. Next, a synthesis tool is used to generate detailed implementations of digital microfluidic biochips from the sequencing graph model. A microfluidic module library is also provided as an input of the synthesis procedure. This module library, analogous to a standard/custom cell library used in cellbased VLSI design, includes different microfluidic functional modules, such as mixers and storage units. Each module is characterized by its function (mixing, storing, detection, etc.) and parameters such as width, length, and operation duration. The microfluidic modules can be characterized through experiments, and their parameters can be stored for use by CAD tools that support large-scale biochip design. In addition, some design specifications are also given a priori, e.g., an upper limit on the completion time, an upper limit on the size of microfluidic array, and the set of nonreconfigurable resources such as on-chip reservoirs/dispensing ports and integrated optical detectors.

The proposed synthesis tool performs both architecturallevel synthesis (e.g., scheduling and resource binding) and geometry-level synthesis (e.g., module placement and routing); its details will be discussed in the next section [40], [41]. The output of the synthesis tools includes the mapping of assay operation to on-chip resources, a schedule for the assay operations, and a 2-D biochip physical design (e.g., the placement of the modules). The synthesis procedure attempts to find a desirable design point that satisfies the input specifications and also optimizes some figures of merit, such as performance and area. Moreover, since digital microfluidics-based biochips need to be tested adequately not only after fabrication, but also continuously during in-field operation, self-testing plays 


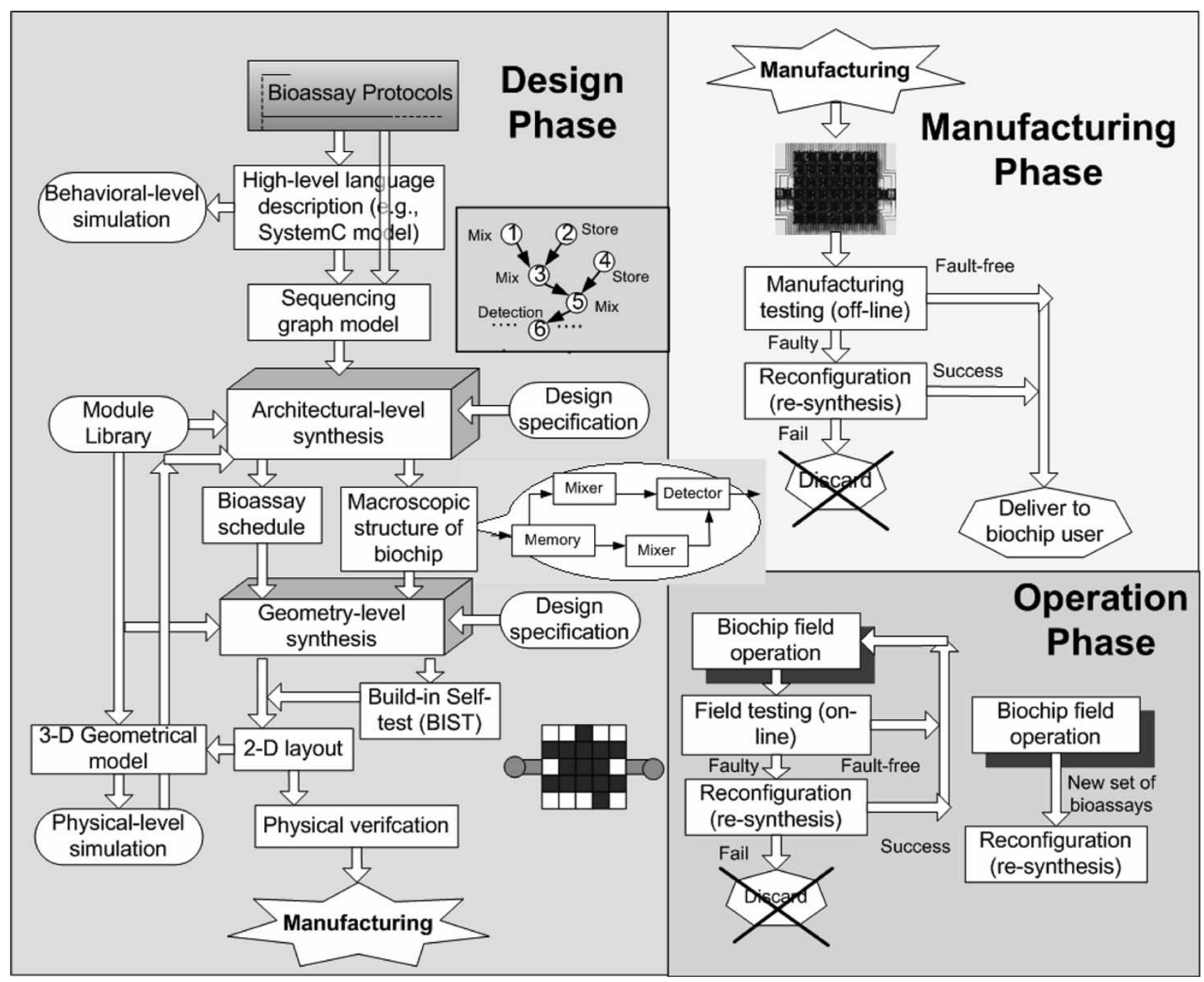

Fig. 11. Overview of top-down design methodology.

an important role in yield enhancement and reliability. Thus, design-for-test (DFT) is also incorporated in the proposed synthesis procedure, whereby a test plan and a set of test hardware (e.g., test droplet sources/sinks and capacitive detection circuits) associated with the synthesized-assay operation and biochip physical design are generated [42], [43]. After synthesis, the 2-D physical design of biochip (i.e., module placement and routing) can be coupled with detailed physical information from a module library (associated with some fabrication technology) to obtain a 3-D geometrical model. This model can be used to perform physical-level simulation and design verification at the low level. After physical verification, a digital microfluidics-based biochip design can be sent for manufacturing.

Digital microfluidics-based biochips are fabricated using standard microfabrication techniques. Due to the underlying mixed technology and multiple energy domains, they exhibit unique failure mechanisms and defects. A manufactured microfluidic array may contain several defective components. We have observed defects such as dielectric breakdown, shorts between adjacent electrodes, and electrode degradation; details are shown in Section III-B3. Reconfiguration techniques can be used to bypass faulty components to tolerate manufacturing defects. Bioassay operations bound to these faulty resources in the original design need to be remapped to other fault-free resources. Due to the strict resource constraints in the fabricated biochip, alterations in the resource-binding operation, schedule, and physical design must be carried out carefully. Our proposed system-level synthesis tool can be easily modified to deal with the reconfiguration issue to support defect tolerance. Using the enhanced synthesis tool, a set of bioassays can be easily mapped to a biochip with a few defective unit cells. Thus, we do not need to discard the defective biochip, thereby leading to higher yield.

As digital microfluidics-based biochips are widely deployed in safety-critical applications, field testing is also required to ensure the high reliability of biochips. Once the testing procedure determines the faulty status of biochips, the operation of the normal bioassay is stopped. Then, reconfiguration techniques are applied to tolerate operational faults; the biochip is redesigned with the help of the proposed system-level designautomation tools. In addition, the similar reconfiguration and design-automation techniques can also be applied to remap a new set of bioassays to a fabricated microfluidic biochip, 
thereby increasing resource utilization and reducing the manufacturing cost.

Compared to the full-custom and bottom-up design methods, this top-down system-level design methodology not only reduces the design-cycle time and time-consuming redesign efforts, but it can also deal with DFT and design-for-reliability (DFR) issues efficiently. Some important details of this systemlevel design methodology are discussed below.

2) Synthesis Techniques: As more bioassays are executed concurrently on a digital microfluidics-based biochip, system integration and design complexity are expected to increase steadily. Thus, system-level design-automation tools, e.g., synthesis tools, are needed to handle design complexity. Synthesis research for digital microfluidic biochips can benefit from classical CAD techniques, which is a well-studied problem and advances in synthesis techniques for integrated circuits continue even today [44], [45].

We envisage that the synthesis of a digital microfluidic biochip can be divided into two major phases, referred to as architectural-level synthesis (i.e., high-level synthesis) and geometry-level synthesis (i.e., physical design) [40], [41]. A behavioral model for a biochemical assay is first obtained from the protocol for that assay. Next, architectural-level synthesis is used to generate a macroscopic structure of the biochip; this structure is analogous to a structural register-transfer-level (RTL) model in electronic CAD. This macroscopic model provides an assignment of assay functions to biochip resources, as well as a mapping of assay functions to time steps, based in part on the dependencies between them. Finally, geometry-level synthesis creates a physical representation at the geometrical level, i.e., the final layout of the biochip, consisting of the configuration of the microfluidic array, locations of reservoirs and dispensing ports, and other geometric details.

The goal of a synthesis procedure is to select a design that minimizes a certain cost function under resource constraints. For example, architectural-level synthesis for microfluidic biochips can be viewed as the problem of scheduling assay functions and binding them to a given number of resources so as to maximize parallelism, thereby decreasing response time. On the other hand, geometry-level synthesis addresses the placement of resources and the routing of droplets to satisfy objectives such as area or throughput. Defect/fault tolerance can also be included as a critical objective in the proposed synthesis method.

In architectural-level synthesis, both the resource-binding problem and the scheduling problem are addressed to generate a structural view of biochip design. As in the case of highlevel synthesis for integrated circuits, resource binding in the biochip synthesis flow refers to the mapping from bioassay operations to available functional resources. Note that there may be several types of resources for any given bioassay operation. For example, a $2 \times 2$-array mixer, a $2 \times 3$-array mixer, and a $2 \times 4$-array mixer can be used for a droplet mixing operation. In such cases, a resource-selection procedure must be used. On the other hand, due to the resource constraints, a resource binding may associate one functional resource with several assay operations; this necessitates resource sharing. Once resource binding is carried out, the time duration for each bioassay operation can be easily determined. Scheduling determines the start times and stop times of all assay operations, subject to the precedence constraints imposed by the sequencing graph. In a valid schedule, assay operations that share a microfluidic module cannot execute concurrently. We have developed an optimal strategy based on integer linear programming for scheduling assay operations under resource constraints [40]. Since the scheduling problem is NP-complete, we have also developed two heuristic techniques that scale well for large problem instances. While the heuristic based on list scheduling is computationally more efficient, the second heuristic based on genetic algorithms yields lower completion times for bioassays. In addition, the heuristic based on genetic algorithms is also able to handle resource binding. Experiments show that the results obtained from the heuristics are close to the provable lower bound for a bioassay of large size [40].

A key problem in the geometry-level synthesis of biochips is the placement of microfluidic modules such as different types of mixers and storage units. Based on the results obtained from architectural-level synthesis (i.e., a schedule of bioassay operation, a set of microfluidic modules, and the binding of bioassay operations to modules), placement determines the locations of each module on the microfluidic array in order to optimize some design metrics. Since digital microfluidics-based biochips enable dynamic reconfiguration of the microfluidic array during run time, they allow the placement of different modules on the same location during different time intervals. Thus, the placement of modules on the microfluidic array can be modeled as a 3-D packing problem. Each microfluidic module is represented by a 3-D box, the base of which denotes the rectangular area of the module and the height denotes the time span of its operation. The microfluidic-biochip placement can now be viewed as the problem of packing these boxes to minimize the total base area, while avoiding overlaps. Since the placement problem is known to be NP-complete [44], a simulated annealing-based heuristic approach has been developed to solve the problem in a computationally efficient manner [41]. Solutions for the placement problem can provide the designer with guidelines on the size of the array to be manufactured. If module placement is carried out for a fabricated array, area minimization frees up more unit cells for sample collection and preparation.

3) Testing Techniques and DFT: Over the past decade, the focus in testing research has broadened from logic and memory tests to include the testing of analog and mixed-signal circuits. Compared to the relatively mature integrated-circuit testing field, MEMS testing is still in its infancy. Recently, fault modeling and fault simulation in surface micromachined MEMS has received attention [46], [47]. However, test techniques for MEMS cannot be directly applied to microfluidics-based biochips, since the techniques and tools currently in use for MEMS testing do not handle fluids. Recently, fault modeling, fault simulation, and a DFT methodology for continuous-flow microfluidic systems have been proposed [48]-[50]. Although advances in test technology are now required to facilitate the continued growth of composite microfluidic systems based on droplet flow, very limited work on the testing for such "digital" microfluidic biochips has been reported to date. 


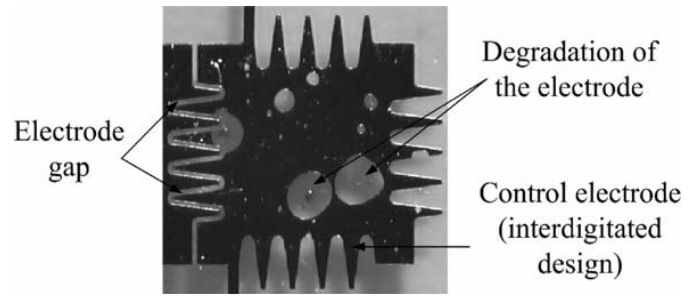

Fig. 12. Top view of a faulty unit cell: Electrode degradation.

We can classify the faults in these systems as being either catastrophic or parametric, along the line of fault classification for analog circuits [51]. Catastrophic (hard) faults lead to a complete malfunction of the system, while parametric (soft) faults cause a deviation in the system performance. A parametric fault is detectable only if this deviation exceeds the tolerance in system performance. Due to their underlying mixed technology and multiple energy domains, digital microfluidicsbased biochips exhibit failure mechanisms and defects that are significantly different from the failure modes in integrated circuits.

Catastrophic faults in digital microfluidics-based biochips may be caused by the following physical defects.

1) Dielectric breakdown: The breakdown of the dielectric at high voltage levels creates a short between the droplet and the electrode. When this happens, the droplet undergoes electrolysis, thereby preventing further transportation.

2) Short between the adjacent electrodes: If a short occurs between two adjacent electrodes, the two electrodes shorted effectively form one longer electrode. When a droplet resides on this electrode, it is no longer large enough to overlap the gap between adjacent electrodes. As a result, the actuation of the droplet can no longer be achieved.

3) Degradation of the insulator: This degradation effect is unpredictable and may become apparent gradually during the operation of the microfluidic system. Fig. 12 illustrates the electrode degradation due to the insulator degradation defect [26]. A consequence of insulator degradation is that droplets often fragment and their motion is prevented because of the unwanted variation of surface-tension forces along their flow path.

4) Open in the metal connection between the electrode and the control source: This defect results in a failure in activating the electrode for transport.

Physical defects that cause parametric faults include the following.

1) Geometrical-parameter deviation: The deviation in insulator thickness, electrode length, and height between parallel plates may exceed their tolerance value.

2) Change in viscosity of droplet and filler medium. These deviations can occur during operation due to an unexpected biochemical reaction, or changes in operational environment, e.g., temperature variation.

Faults in microfluidics-based biochips can also be classified based on the time at which they appear. Therefore, system failure or degraded performance can either be caused by manu- facturing defects or they might be due to parametric variations. Testing of manufacturing defects, such as a short between the adjacent electrodes or a deviation in the value of the geometrical parameters, should be performed immediately after production. However, operational faults, such as degradation of the insulator or change in fluid viscosity, can occur throughout the lifetime of the system. Therefore, concurrent testing during system operation is necessary for such faults.

We have proposed a unified test methodology for digital microfluidic biochips, whereby faults can be detected by controlling and tracking droplet motion electrostatically [52], [53]. Based on this unified detection mechanism, we can dispense the test-stimuli droplet containing the normal conductive fluid (e.g., $\mathrm{KCl}$ solution) into the microfluidic system under test from the droplet source. These droplets are guided through the unit cells following the test plan towards the droplet sink, which is connected to an integrated capacitive detection circuit. Most catastrophic faults result in a complete cessation of droplet transportation [52], [53]. Thus, for the faulty system, the teststimuli droplet is stuck during its motion. On the other hand, for the fault-free system, all the test-stimuli droplets can be observed at the droplet sink by the capacitive detection circuit. Therefore, we can easily determine the fault-free or faulty status of the droplet-based microfluidic system by simply observing the arrival of test-stimuli droplets at some selected ports of the system.

An efficient test plan not only ensures that the testing operation does not conflict with the normal biomedical assay, but it also guides test-stimuli droplets to cover all the unit cells available for testing. This test plan can be optimized to minimize the total-testing-time cost for a given test hardware overhead, which refers here to the number of droplet sources and droplet sinks. We can formulate the test planning problem in terms of the graph partitioning and the Hamiltonian path problems from graph theory [42]. Since this optimization problem can be proven to be NP-complete, we also develop heuristic approaches to solve the test planning problem [42]. Experimental results indicate that for large array sizes, heuristic methods yield solutions close to provable lower bounds while ensuring scalability and low computation cost.

The proposed testing methodology can be used for the field testing of digital microfluidics-based systems; as a result, it increases the system reliability during everyday operation [43]. With negligible hardware overhead, this method also offers an opportunity to implement BIST for microfluidic systems and therefore eliminate the need for costly, bulky, and expensive external test equipment. Furthermore, after detection, droplet flow paths for biomedical assays can be reconfigured dynamically such that faulty unit cells are bypassed without interrupting the normal operation. Thus, this approach increases fault tolerance and system lifetime when such systems are deployed for safetycritical applications.

4) Reconfiguration Techniques and DFR: As in the case of integrated circuits, increase in the density and area of microfluidic biochips may reduce yield, especially for smaller feature sizes. It will take time to ramp up the yield based on an understanding of defects in such biochips. Therefore, defect tolerance for digital microfluidic biochips is especially important 
for the emerging marketplace. Moreover, some manufacturing defects are expected to be latent and they may manifest themselves during field operation of the biochips. Since many microfluidic biochips are intended for safety-critical applications, system dependability is an essential performance parameter. Thus, fault-tolerance techniques will play a critical role in field applications, especially in harsh operational environments. Efficient reconfiguration techniques are motivated by the need for defect/fault tolerance.

A digital microfluidics-based biochip can be viewed as a dynamically reconfigurable system. If a unit cell becomes faulty during the operation of the biochip, and the fault is detected using the proposed testing technique, the microfluidic module containing this unit cell can easily be relocated to another part of the microfluidic array by changing the control voltages applied to the corresponding electrodes. Fault-free unused unit cells in the array are utilized to accommodate the faulty module. Hence, the configuration of the microfluidic array, i.e., the placement of the microfluidic modules, influences the faulttolerance capability of the biochip. Thus, we introduce a simple measure, referred to as the fault-tolerance index, to evaluate the fault-tolerance capability of the microfluidic biochip; this measure is incorporated into the placement procedure [41]. This DFR procedure leads to a small biochip area due to the efficient utilization of dynamic reconfigurability, as well as high fault tolerance due to the efficient use of spare unit cells.

Defect/fault tolerance can also be achieved by including redundant elements in the system; these elements can be used to replace faulty elements through reconfiguration techniques [54]. Another method is based on graceful degradation, in which all elements in the system are treated in a uniform manner, and no element is designated as a spare [55]. In the presence of defects, a subsystem with no faulty element is first determined from the faulty system. This subsystem provides the desired functionality, but with a gracefully degraded level of performance (e.g., longer execution times). Due to the dynamic reconfigurability of digital microfluidics-based biochips, the microfluidic components (e.g., mixers) used during the bioassay can be viewed as reconfigurable virtual devices. For example, a $2 \times 4$ array mixer (implemented using a rectangular array of control electrodes-two in the $X$-direction and four in $Y$-direction) can easily be reconfigured to a $2 \times 3$ array mixer or a $2 \times 2$ array mixer. This feature facilitates the use of graceful degradation to achieve defect tolerance in digital biochips. Since a high-level scheme is required to efficiently reconfigure and reallocate the assay operations, our proposed system-level design-automation tools can be utilized to support defect/fault tolerance, thereby leading to a high system reliability.

\section{Challenges}

A number of open problems remain to be tackled in the development of the proposed top-down system-level design methodology. First, we note that, following the geometrylevel synthesis, the automatically generated layout of digital microfluidics-based biochips need to be coupled with more detailed geometrical data for 3-D physical simulation. Although this detailed simulation-based approach can be used for phys- ical verification, it is time consuming and highly dependent on the accuracy of the geometrical model. We can speed up and automate the physical-verification procedure for biochip designs by leveraging classical integrated-circuit verification techniques (e.g., design-rule checking). As in circuit design, the layered microfabrication process information can be encapsulated in a layout design-rule file. The synthesized layout of microfluidic biochip is verified to satisfy an abstraction of geometric design constraints, which consequently ensures robust manufacturing. However, the design rules that need to be checked in the microfluidics-based biochips are significantly different from those in circuit area. They are also unlike classical MEMS due to the fluidic domain [56]. The determination of accurate and efficient design rules for the physical verification of digital microfluidics-based biochips remains an open problem.

Effective testing of biochips also needs to be investigated. Some physical-failure mechanisms are not yet well understood. For example, due to the unknown thermal effects on microfluidic-assay operation, the defects associated with power supply or environmental-temperature variation are hard to detect. Efficient fault models and test-stimuli-generation techniques are required for the testing of biochips. Moreover, while catastrophic faults have the highest priority for detection as they result in complete malfunction, parametric faults are much harder to detect and may result in malfunction depending on the application domain and specification. As a result, DFT techniques to handle parametric faults are more complicated than those for the detection of catastrophic faults.

Coupling of energy domains also affect the synthesis and performance optimization of biochips. Due to coupling between different energy domains (e.g., electrical, fluidic, and thermal domains) [2], multiple-objective optimization problems must be solved during synthesis. For example, we should not only aim to minimize the assay operation time, but we should also keep the power consumption low to avoid fluid overheating. Such optimization problems that span several energy domains appear to be extremely difficult. Efficient solutions to such an optimization problem are nevertheless essential to ensure the quality of biochips designed using automated synthesis techniques.

\section{CONCLUSION}

We have presented a new system-level design-automation methodology for droplet-based microfluidic biochips. Technology issues underlying biochips and microfluidics have first been reviewed. We focused here on a new implementation platform for digital microfluidic biochips based on EWOD. The level of system integration and the complexity of digital microfluidicsbased biochips are expected to increase in the near future due to the growing need for multiple and concurrent bioassays on a chip. To address the associated design challenges, we have proposed a top-down design methodology for digital microfluidic biochips. In this proposed method, synthesis tools are used to map the behavioral description of bioassays to a microfluidic biochip and generate an optimized schedule of bioassay operations, the binding of assay operations to 
resources, and a layout of the microfluidic biochip. Compared to the current full-custom and bottom-up design methods, this top-down system-level design methodology not only reduces the design cycle time and time-consuming redesign efforts, but it can also deal with DFT and DFR issues efficiently. For fabricated microfluidic biochips, cost-effective testing techniques have been proposed to detect faulty unit cells after manufacturing and during field operation. Dynamic reconfiguration techniques, incorporated in these design-automation tools, are also used to easily bypass faulty unit cells once they are detected, and remap bioassays operations to other fault-free resources, thereby supporting defect/fault tolerance. This work is expected to reduce human efforts and enable high-volume production and applications of microfluidics-based biochips, thereby paving the way for the integration of biochip components in the next generation of SOC designs, as envisaged by the 2003 ITRS document.

\section{REFERENCES}

[1] M. A. Burns et al., "An integrated nanoliter DNA analysis device," Science, vol. 282, no. 5388, pp. 484-487, Oct. 1998.

[2] T. Zhang, K. Chakrabarty, and R. B. Fair, Microelectrofluidic Systems: Modeling and Simulation. Boca Raton, FL: CRC Press, 2002.

[3] T. Thorsen, S. Maerkl, and S. Quake, "Microfluidic large-scale integration," Science, vol. 298, no. 5593, pp. 580-584, Oct. 2002.

[4] E. Verpoorte and N. F. De Rooij, "Microfluidics meets MEMS," Proc. IEEE, vol. 91, no. 6, pp. 930-953, Jun. 2003.

[5] T. H. Schulte, R. L. Bardell, and B. H. Weigl, "Microfluidic technologies in clinical diagnostics," Clin. Chim. Acta, vol. 321, no. 1-2, pp. 1-10, Jul. 2002.

[6] V. Srinivasan, V. K. Pamula, and R. B. Fair, "An integrated digital microfluidic lab-on-a-chip for clinical diagnostics on human physiological fluids," Lab Chip, vol. 4, no. 4, pp. 310-315, Aug. 2004.

[7] H. F. Hull, R. Danila, and K. Ehresmann, "Smallpox and bioterrorism: Public-health responses," J. Lab. Clin. Med., vol. 142, no. 4, pp. 221-228, Oct. 2003.

[8] S. Venkatesh and Z. A. Memish, "Bioterrorism: A new challenge for public health," Int. J. Antimicrob. Agents, vol. 21, no. 2, pp. 200-206, Feb. 2003.

[9] M. G. Pollack, R. B. Fair, and A. D. Shenderov, "Electrowetting-based actuation of liquid droplets for microfluidic applications," Appl. Phys. Lett., vol. 77, no. 11, pp. 1725-1726, Sep. 2000.

[10] S. K. Cho, S. K. Fan, H. Moon, and C. J. Kim, "Toward digital microfluidic circuits: Creating, transporting, cutting and merging liquid droplets by electrowetting-based actuation," in Proc. IEEE Micro Electro Mechanical Systems (MEMS) Conf., Las Vegas, NV, 2002, pp. 32-52.

[11] International Technology Roadmap for Semiconductors (ITRS). [Online]. Available: http://public.itrs.net/Files/2003ITRS/Home2003.htm

[12] Affymetrix GeneChip. [Online]. Available: http://www.affymetrix.com

[13] Infineon Electronic DNA Chip. [Online]. Available: http://www. infineon.com

[14] Nanogen NanoChip. [Online]. Available: http://www.nanogen.com

[15] S. Mutlu et al., "Enhanced electro-osmosis pumping with liquid bridge and field effect flow rectification," in Proc. IEEE Micro Electro Mechanical Systems (MEMS) Conf., Maastricht, The Netherlands, 2004, pp. $850-853$.

[16] B. S. Gallardo et al., "Electrochemical principles for active control of liquids on submillimeter scales," Science, vol. 283, no. 5398, pp. 57-60, Jan. 1999.

[17] K. Ichimura, S. Oh, and M. Nakagawa, "Light-driven motion of liquids on a photoresponsive surface," Science, vol. 288, no. 5471, pp. 1624-1626, Jun. 2000.

[18] T. S. Sammarco and M. A. Burns, "Thermocapillary pumping of discrete droplets in microfabricated analysis devices," AIChe J., vol. 45, no. 2, pp. 350-366, 1999.

[19] G. N. Somero, "Proteins and temperature," Аnпи. Rev. Physiol., vol. 57, pp. 43-68, 1995

[20] A. Wixforth, and J. Scriba, Nanopumps for Programmable Biochips. [Online]. Available: http://www.advalytix.de
[21] M. Washizu, "Electrostatic actuation of liquid droplets for microreactor applications," IEEE Trans. Ind. Appl., vol. 34, no. 4, pp. 732-737, Jul./Aug. 1998.

[22] T. B. Jones et al., "Dielectrophoretic liquid actuation and nanodroplet formation," J. Appl. Phys., vol. 89, no. 3, pp. 1441-1448, Feb. 2001.

[23] J. Vykoukal et al., "A programmable dielectric fluid processor for dropletbased chemistry," in Micro Total Analysis Systems, Monterey, CA, 2001, pp. 72-74.

[24] A DEP Primer. [Online]. Available: http://www.dielectrophoresis.org

[25] T. B. Jones, K. L. Wang, and D. J. Yao, "Frequency-dependent electromechanics of aqueous liquids: Electrowetting and dielectrophoresis," Langmuir, vol. 20, no. 7, pp. 2813-2818, Mar. 2004.

[26] M. Pollack, "Electrowetting-based microactuation of droplets for digital microfluidics," Ph.D. thesis, Dept. Elect. Comput. Eng., Duke Univ., Durham, NC, 2001.

[27] M. G. Pollack, A. D. Shenderov, and R. B. Fair, "Electrowetting-based actuation of droplets for integrated microfluidics," Lab Chip, vol. 2, no. 1, pp. 96-101, 2002.

[28] V. Srinivasan, V. K. Pamula, M. G. Pollack, and R. B. Fair, "A digital microfluidic biosensor for multianalyte detection," in Proc. IEEE Micro Electro Mechanical Systems (MEMS) Conf., Kyoto, Japan, 2003, pp. 327-330.

[29] - "Clinical diagnostics on human whole blood, plasma, serum, urine, saliva, sweat, and tears on a digital microfluidic platform," in Proc. $\mu T A S$, Squaw Valley, CA, 2003, pp. 1287-1290.

[30] V. Srinivasan, "A digital microfluidic lab-on-a-chip for clinical diagnostic applications," Ph.D. thesis, Dept. Elect. Comput. Eng., Duke Univ., Durham, NC, 2005.

[31] S. Senturia, "Microfabricated structures for the measurement of mechanical properties and adhesion of thin films," in Proc. Int. Conf. Solid-State Sensors and Actuators (Transducers), Tokyo, Japan, 1987, pp. 11-16.

[32] G. K. Fedder and Q. Jing, "A hierarchical circuit-level design methodology for microelectromechinal system," IEEE Trans. Circuits Syst. II, Analog Digit. Signal Process., vol. 46, no. 10, pp. 1309-1315, Oct. 1999.

[33] S. K. De and N. R Aluru, "Physical and reduced-order dynamic analysis of MEMS," in Proc. IEEE/ACM Int. Conf. Computer-Aided Design, San Jose, CA, 2003, pp. 270-273.

[34] T. Mukherjee and G. K. Fedder, "Design methodology for mixed-domain systems-on-a-chip [MEMS design]," in Proc. IEEE VLSI System Level Design, Orlando, FL, 1998, pp. 96-101.

[35] A. B. Kahng et al., "Evaluation of placement techniques for DNA probe array layout," in Proc. IEEE/ACM Int. Conf. Computer-Aided Design, San Jose, CA, 2003, pp. 262-269.

[36] A. N. Chatterjee and N. R. Aluru, "Combined circuit/device modeling and simulation of integrated microfluidic systems," J. Microelectromech. Syst., vol. 14, no. 1, pp. 81-95, Feb. 2005.

[37] B. Shapiro et al., "Modeling of electrowetted surface tension for addressable microfluidic systems: Dominant physical effects, material dependences, and limiting phenomena," in Proc. IEEE Micro Electro Mechanical Systems (MEMS) Conf., Kyoto, Japan, 2003, pp. 201-205.

[38] J. Zeng and F. T. Korsmeyer, "Principles of droplet electrohydrodynamics for lab-on-a-chip," Lab Chip, vol. 4, no. 4, pp. 265-277, 2004.

[39] CoventorWare. [Online]. Available: http://www.coventor.com

[40] F. Su and K. Chakrabarty, "Architectural-level synthesis of digital microfluidics-based biochips," in Proc. IEEE Int. Conf. Computer-Aided Design, San Jose, CA, 2004, pp. 223-228.

[41] _ - "Design of fault-tolerant and dynamically-reconfigurable microfluidic biochips," in Proc. Design, Automation and Test Eur. (DATE) Conf., Munich, Germany, 2005, pp. 1202-1207.

[42] F. Su, S. Ozev, and K. Chakrabarty, "Test planning and test resource optimization for droplet-based microfluidic systems," in Proc. Eur. Test Symp., Corsica, France, 2004, pp. 72-77.

[43] _ "Concurrent testing of droplet-based microfluidic systems for multiplexed biomedical assays," in Proc. IEEE Int. Test Conf., Charlotte, NC, 2004, pp. 883-892.

[44] G. De Micheli, Synthesis and Optimization of Digital Circuits. New York: McGraw-Hill, 1994.

[45] R. Camposano, "Behavioral synthesis," in Proc. IEEE/ACM Design Automation Conf., Las Vegas, NV, 1996, pp. 33-34.

[46] A. Kolpekwar and R. D. Blanton, "Development of a MEMS testing methodology," in Proc. Int. Test Conf., Washington, DC, 1997, pp. 923-937.

[47] N. Deb and R. D. Blanton, "Analysis of failure sources in surfacemicromachined MEMS," in Proc. Int. Test Conf., Atlantic City, NJ, 2000, pp. 739-749. 
[48] H. G. Kerkhoff, "Testing philosophy behind the micro analysis system," in Proc. SPIE, Paris, France, 1999, vol. 3680, pp. 78-83.

[49] H. G. Kerkhoff and H. P. A. Hendriks, "Fault modeling and fault simulation in mixed micro-fluidic microelectronic systems," J. Electron. Test.: Theory Appl., vol. 17, no. 5, pp. 427-437, Oct. 2001.

[50] H. G. Kerkhoff and M. Acar, "Testable design and testing of microelectro-fluidic arrays," in Proc. IEEE VLSI Test Symp., Napa, CA, 2003, pp. 403-409.

[51] A. Jee and F. J. Ferguson, "Carafe: An inductive fault analysis tool for CMOS VLSI circuits," in Proc. IEEE VLSI Test Symp., Atlantic City, NJ, 1993, pp. 92-98.

[52] F. Su, S. Ozev, and K. Chakrabarty, "Testing of droplet-based microelectrofluidic systems," in Proc. IEEE Int. Test Conf., Charlotte, NC, 2003, pp. 1192-1200.

[53] idic biosensor systems," IEEE Sensors J., vol. 5, no. 4, pp. 763-773, Aug. 2005.

[54] F. Su, K. Chakrabarty, and V. K. Pamula, "Yield enhancement of digital microfluidics-based biochips using space redundancy and local reconfiguration," in Proc. Design Automation and Test in Europe (DATE) Conf., Munich, Germany, 2005, pp. 1196-1201.

[55] F. Su and K. Chakrabarty, "Defect tolerance for gracefully-degradable mocrofluidics-based chips," in Proc. IEEE VLSI Test Symp., Palm Springs, CA, 2005, pp. 321-326.

[56] T. Mukherjee, "MEMS design and verification," in Proc. IEEE Int. Test Conf., Charlotte, NC, 2003, pp. 681-690.

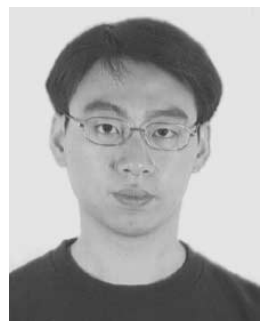

Fei Su (S'03) received the B.E. and M.S. degrees in automation from Tsinghua University, Beijing, China, in 1999 and 2001, respectively, and the M.S. degree in electrical and computer engineering from Duke University, Durham, NC, in 2003. He is now pursuing the Ph.D. degree in electrical and computer engineering at Duke University.

$\mathrm{He}$ is interested in research on the design and testing of mixed-technology microsystems, electronic design automation, mixed-signal very-large-scale integration (VLSI) design, microelectromechanical system (MEMS) modeling, and simulation.

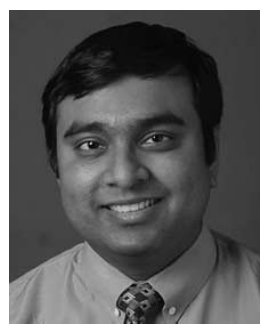

Krishnendu Chakrabarty (S'93-M'96-SM'00) received the B.Tech. degree from the Indian Institute of Technology, Kharagpur, India, in 1990, and the M.S.E. and Ph.D. degrees from the University of Michigan, Ann Arbor, in 1992 and 1995, respectively, all in computer science and engineering.

$\mathrm{He}$ is now an Associate Professor of Electrical and Computer Engineering at Duke University. His current research projects include the following: design and testing of system-on-chip integrated circuits; design automation of microfluidics-based biochips; microfluidics-based chip cooling; distributed sensor networks; embedded realtime systems. He is a coauthor of two books, namely Microelectrofluidic Systems: Modeling and Simulation (Boca Raton, FL: CRC Press, 2002) and Test Resource Partitioning for System-on-a-Chip (Norwell, MA: Kluwer, 2002), and the Editor of SOC (System-on-a-Chip) Testing for Plug and Play Test Automation (Norwell: Kluwer, MA, 2002). He is also a coauthor of the forthcoming book Scalable Infrastructure for Distributed Sensor Networks (London: Springer). He has published 200 papers in journals and refereed conference proceedings, and he holds a U.S. patent in built-in self-test. He is an Associate Editor of the ACM Journal on Emerging Technologies in Computing Systems, an Editor of the Journal of Electronic Testing: Theory and Applications (JETTA), and a Member of the editorial board for Sensor Letters and the Journal of Embedded Computing. He serves as a Subject Area Editor for the International Journal of Distributed Sensor Networks.
Dr. Chakrabarty is a member of ACM, ACM SIGDA, and Sigma Xi. He is a recipient of the National Science Foundation Early Faculty (CAREER) Award and the Office of Naval Research Young Investigator Award. He is also a recipient of best paper awards at the 2005 IEEE International Conference on Computer Design and the 2001 IEEE Design, Automation and Test in Europe (DATE) Conference. He is also a recipient of the Humboldt Research Fellowship, awarded by the Alexander von Humboldt Foundation, Germany. $\mathrm{He}$ is an Associate Editor of the IEEE TRANSACTIONS ON COMPUTER-AIDED DESIGN OF INTEGRATED CIRCUITS AND SYSTEMS and an Associate Editor of the IEEE TRANSACTIONS ON VERY LARGE SCALE INTEGRATION SYSTEMS. He has also served as an Associate Editor of the IEEE TRANSACTIONS ON Circuits and Systems II: ANAlog and Digital Signal Processing. He serves as the Vice Chair of Technical Activities in IEEE's Test Technology Technical Council and is a Member of the program committees of several IEEE/ACM conferences and workshops. He chaired the emerging technologies subcommittee for the IEEE International Conference on CAD, 2005, and he is designated as a Subcommittee Chair for new, emerging, and specialized technologies for the 2006 Design Automation Conferences. He served as a Tutorial Co-Chair for the 2005 IEEE International Conference on VLSI Design, and is the designated Program Co-Chair for the 2005 IEEE Asian Test Symposium.

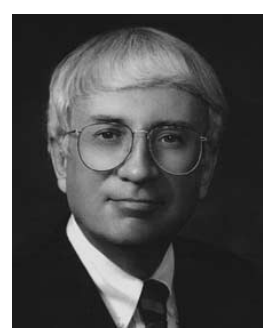

Richard B. Fair (S'63-M'75-SM'75-F'90) received the Ph.D. degree from Duke University, Durham, NC, in 1969.

He spent 12 years at Bell Labs working on semiconductor devices and integrated-circuit technology. He returned to North Carolina in 1981 and spent 13 years as a Vice President of Microelectronics Center of North Carolina (MCNC), having responsibilities in chip design, computer-aided design, packaging, process technology, and microelectromechanical system (MEMS). He is currently a Professor of electrical and computer engineering at Duke University. He has published over 150 papers in refereed journals and conference proceedings, written ten book chapters, edited nine books or conference proceedings, and given over 120 invited talks, mostly in the area of semiconductor devices or the fabrication thereof. His current research interests include bio-MEMS and digital microfluidic chips. His research group first demonstrated microdroplet transport over electrode arrays based on electrowetting actuation.

Dr. Fair is a Fellow of the Electrochemical Society, past Editor-in-Chief of the PROCEEDINGs OF THE IEEE, and he has served as Associate Editor of the IEEE TRANSACTIONS ON ELECTRON DEVICES. He is a recipient of the IEEE Third Millennium Medal (2000) and the 2003 Solid State Science and Technology Prize and Medal from the Electrochemical Society, which was presented in Paris, France. 